1967, Bull. Astr. Inst. Netherlands 19 107-112; Communication from the Observatory at Leiden

\title{
HIGH-VELOCITY CLOUD COLLISIONS
}

\author{
M. P. SAVEDOFF*, J. W. HOVENIER AND B. VAN LEER
}

Received 23 November 1966

High-velocity cloud collisions may occur when extragalactic gas penetrates the Galaxy, as suggested by OORT (1966). At a relative velocity of $500 \mathrm{~km} / \mathrm{sec}$ shocks will occur at heights of at most $1 \mathrm{kpc}$ above the galactic plane. We first describe a model in which ionizing shocks of constant strength run from the separation surface both into galactic and extragalactic gas (section 2). The time it takes the ionized gases to cool down and become neutral

\section{Introduction}

Recent 21-cm observations at Dwingeloo Radio Observatory by HuLsBosCH and RAIMOND (1966) have revealed the presence of thirty gas clouds with high negative velocities (typically $-120 \mathrm{~km} / \mathrm{sec}$ with respect to the local standard of rest). Among other possibilities, OORT (1966) has suggested that these features may result from the impact of inflowing extragalactic gas on galactic gas, the extragalactic clouds having initial velocities of about $-500 \mathrm{~km} / \mathrm{sec}$ with respect to the local standard of rest. This paper is a preliminary attempt to describe the braking and accretion mechanism.

A hydrogen atom with a velocity of $v \mathrm{~km} / \mathrm{sec}$ has a kinetic energy of $0.0052 v^{2} \mathrm{eV}$. Hence the energy dissipated in the clouds penetrating the corona must be of the order of $0.0052 \times\left(500^{2}-120^{2}\right) \approx 1200 \mathrm{eV}$ per particle. It is clear that both extragalactic and galactic gas will be ionized during their interaction, and in order to explain neutral hydrogen at the observed velocities, recombination must occur after the gas has radiated away the surplus energy.

As in neither of the gases the initial conditions are very certain both gases are taken to be originally neutral, at temperatures of about $100{ }^{\circ} \mathrm{K}$. Though far from realistic, one-dimensional steady flow has been used as an approximation of the full hydrodynamic equations.

* University of Rochester, N.Y., U.S.A.; National Science Foundation Senior Postdoctoral Fellow at Leiden 1964-65. and observable in the $21-\mathrm{cm}$ line is then estimated (section 3 ). The preceding results are provisionally combined in a model which yields a surface density of the neutral gas consistent with the observations. The observed high-velocity features may consist of galactic gas, the extragalactic gas being too hot to be seen at the same time.

\section{Shock relations}

As the Mach number of the flow is very high, about 500 , shocks will occur. Generally a shock thickness is some fraction of the mean free path in the shocked gas. The mean free path can be written as $\lambda^{\prime}=\left(\sigma^{\prime} n^{\prime}\right)^{-1}$, where $\sigma^{\prime}$ and $n^{\prime}$ are respectively collision cross-section and total particle density in the ionized gas. As will be shown in section 2.2 the temperature of the shocked gas is typically $1.5 \times 10^{6}{ }^{\circ} \mathrm{K}$, at which temperature the Coulomb cross-section of ionized hydrogen is about $2.0 \times 10^{-17} \mathrm{~cm}^{2}$. If we impose the condition that the shock thickness must not exceed $10 \mathrm{pc}$, to make it at least an order of magnitude smaller than the characteristic size of the gas masses, we find that $n^{\prime}$ must be greater than $1.6 \times 10^{-3} \mathrm{~cm}^{-3}$. This corresponds to an initial hydrogen density $n_{\mathrm{H}}$ exceeding $2.0 \times 10^{-4} \mathrm{~cm}^{-3}$, since for strong shocks $n^{\prime} \approx 2 n_{\mathrm{H}}^{\prime} \approx 8 n_{\mathrm{H}}$.

Little is known about the density of the extragalactic gas. According to a rough formula the density of galactic hydrogen varies with the height above the galactic plane $z$ as $n_{\mathrm{H}}=0.7 \exp (-z / 120) ; z$ expressed in parsecs. Thus it seems unlikely that the present description in terms of discrete shocks is applicable at heights over $1 \mathrm{kpc}$.

The basic hydrodynamic equations for steady flow containing a shock are briefly reviewed in section 2.1 .

\subsection{Single shock}

The equations of motion for one-dimensional steady flow containing a shock are most conveniently written 
as conservation equations in a frame of reference moving along with the shock:

conservation of mass

$$
\rho_{1} V_{1}=\rho_{2} V_{2}
$$

conservation of momentum

$$
P_{1}+\rho_{1} V_{1}^{2}=P_{2}+\rho_{2} V_{2}^{2}
$$

conservation of energy

$$
\frac{\gamma}{\gamma-1} \frac{P_{1}}{\rho_{1}}+\frac{1}{2} V_{1}^{2}=\frac{\gamma}{\gamma-1} \frac{P_{2}}{\rho_{2}}+\frac{1}{2} V_{2}^{2}+\frac{1}{2} V_{i}^{2} .
$$

The notation is the usual; index 1 refers to the neutral medium before the shock and index 2 refers to the ionized medium behind the shock (see figure 1). Ionization has been included by adding $\frac{1}{2} V_{i}^{2}$, the ionization energy per gram, to the internal energy of the shocked gas.

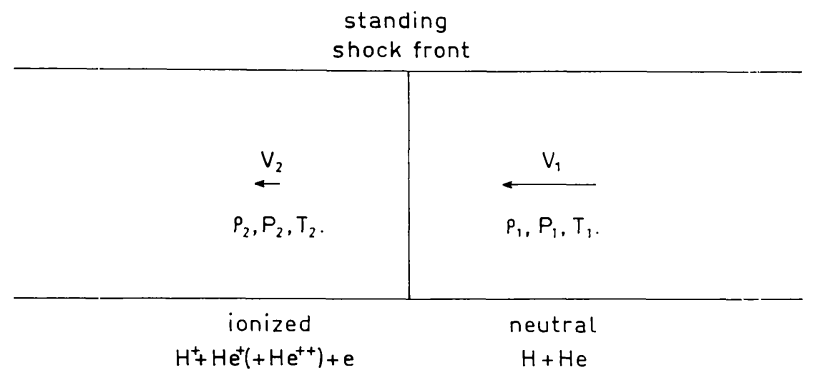

Figure 1. Standing ionizing shock.

In the case considered the initial pressure $P_{1}$ is always very low and may be neglected in eqs. (2) and (3). In this strong shock approximation, elimination of $V_{1}$ and $V_{2}$ from eq. (3) yields

$$
\frac{\rho_{1}}{\rho_{2}}=\frac{\gamma-1}{\gamma+1}\left(1-\frac{\rho_{1}}{P_{2}} V_{i}^{2}\right) \text {. }
$$

With the same accuracy, eqs. (1) and (2) give

$$
P_{2}=\rho_{1} V_{1}^{2}\left(1-\frac{\rho_{1}}{\rho_{2}}\right) \text {. }
$$

Substitution of eq. (5) into eq. (4) leads to a quadratic equation in $\rho_{1} / \rho_{2}$ with solution

$$
\frac{\rho_{1}}{\rho_{2}}=\frac{1}{\gamma+1}\left[\gamma-\sqrt{1+\left(\gamma^{2}-1\right) \frac{V_{i}^{2}}{V_{1}^{2}}}\right] \text {. }
$$

The choice of a minus sign before the square root corresponds to the well known exclusion of an expansion wave by the second law of thermodynamics.

Once $\rho_{1} / \rho_{2}$ has been found, $P_{2} / P_{1}$ can be evaluated from eq. (5) and subsequently $T_{2} / T_{1}$, as

$$
\frac{T_{2}}{T_{1}}=\frac{\mu_{2}}{\mu_{1}} \frac{\rho_{1}}{\rho_{2}} \frac{P_{2}}{P_{1}}
$$

Here $\mu$ denotes mean particle mass expressed in proton masses.

Table 1 summarizes some values of $\mu$ and $V_{i}$. Upper two lines: no helium present, lower four lines: helium abundance 10 per cent of hydrogen abundance by number, with different assumptions about its ionization.

TABLE 1

Some values of the ionization parameters

\begin{tabular}{c|c|l|l|l|l|l|c}
\hline$n_{\mathrm{H}}$ & $n_{\mathrm{H}^{+}}$ & $n_{\mathrm{He}}$ & $n_{\mathrm{He}^{+}}$ & $n_{\mathrm{He}^{++}}$ & $n_{\mathrm{e}}$ & $\mu$ & $\begin{array}{c}V_{i} \\
(\mathrm{~km} / \mathrm{sec})\end{array}$ \\
\hline 1 & 0 & 0 & 0 & 0 & 0 & 1.000 & 0 \\
0 & 1 & 0 & 0 & 0 & 1 & 0.500 & 51 \\
1 & 0 & 0.1 & 0 & 0 & 0 & 1.273 & 0 \\
0 & 1 & 0.1 & 0 & 0 & 1 & 0.667 & 43 \\
0 & 1 & 0 & 0.1 & 0 & 1.1 & 0.636 & 47 \\
0 & 1 & 0 & 0 & 0.1 & 1.2 & 0.609 & 54 \\
\hline
\end{tabular}

In table 2, values of $\rho_{1} / \rho_{2}, P_{2} / P_{1}$ and $T_{2}$ as functions of $V_{1}$ have been calculated from eqs. (6), (5) and (7) using the following data: $\gamma=5 / 3, T_{1}=100{ }^{\circ} \mathrm{K}, \mu_{1}=$ 1.273 (from line 3 of table 1) and $\mu_{2}=0.625, V_{i}=50$ $\mathrm{km} / \mathrm{sec}$ (a case between lines 5 and 6 of table 1). A consistent solution would require that for a given set of values $\rho_{1}, P_{1}, V_{1}$, the values for $\mu_{2}$ and $V_{i}$ are appropriate to the acquired degree of ionization. These conditions can obviously not be fulfilled in the top lines of the table, where $V_{1}$ is still too close to the fixed value of $V_{i}$.

TABLE 2

Shock amplitudes

\begin{tabular}{c|c|c|c}
\hline $\begin{array}{c}V_{1} \\
(\mathrm{~km} / \mathrm{sec})\end{array}$ & $\rho_{1} / \rho_{2}$ & $P_{2} / P_{1}$ & $\begin{array}{c}T_{2} \\
\left({ }^{\circ} \mathrm{K}\right)\end{array}$ \\
\hline 60 & 0.0659 & $5.19 \times 10^{3}$ & $1.68 \times 10^{4}$ \\
75 & 0.1234 & $7.61 \times 10^{3}$ & $4.61 \times 10^{4}$ \\
100 & 0.1745 & $1.27 \times 10^{4}$ & $1.09 \times 10^{5}$ \\
150 & 0.2147 & $2.73 \times 10^{4}$ & $2.87 \times 10^{5}$ \\
200 & 0.2297 & $4.76 \times 10^{4}$ & $5.37 \times 10^{5}$ \\
300 & 0.2409 & $1.05 \times 10^{5}$ & $1.25 \times 10^{6}$ \\
400 & 0.2448 & $1.87 \times 10^{5}$ & $2.24 \times 10^{6}$ \\
500 & 0.2467 & $2.91 \times 10^{5}$ & $3.52 \times 10^{6}$ \\
600 & 0.2477 & $4.18 \times 10^{5}$ & $5.08 \times 10^{6}$ \\
1000 & 0.2492 & $1.16 \times 10^{6}$ & $1.33 \times 10^{7}$ \\
\hline
\end{tabular}




\subsection{Shocks in colliding clouds}

In our description of colliding gas clouds two shocks are demanded by symmetry (see figure 2). This case has been discussed by BURGERs (1951) for non-ionizing shocks. Index 1 now refers to galactic gas whereas index 2 refers to gas of extragalactic origin. All parameters of the ionized gases are primed. In this section, radiation losses in the ionized gases are still left out of consideration.

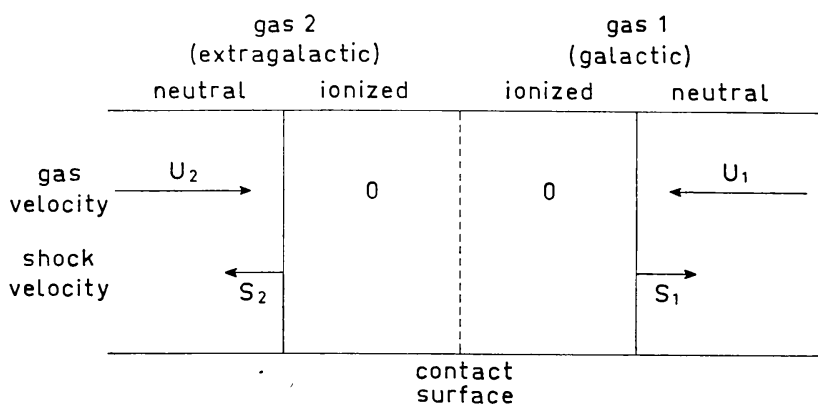

Figure 2. Shocks in colliding gases. The velocities refer to a stationary contact surface. Velocities to the right are positive, hence $U_{1}$ and $S_{2}$ have negative values.

As in section 2.1, the strong shock approximation is used. The density ratios across the shock fronts are taken to be $r_{1}=\rho_{1} / \rho_{1}^{\prime}$ and $r_{2}=\rho_{2} / \rho_{2}^{\prime}$; the various velocities are sufficiently defined in figure 2 . Eqs. (1) and (2) are now replaced by

$$
\begin{aligned}
& r_{1}\left(S_{1}-U_{1}\right)=S_{1} \\
& r_{2}\left(S_{2}-U_{2}\right)=S_{2} \\
& \rho_{1}\left(S_{1}-U_{1}\right)^{2}=P_{1}^{\prime}+\frac{\rho_{1}}{r_{1}} S_{1}^{2} \\
& \rho_{2}\left(S_{2}-U_{2}\right)^{2}=P_{2}^{\prime}+\frac{\rho_{2}}{r_{2}} S_{2}^{2} .
\end{aligned}
$$

If we choose values of $r_{1}$ and $r_{2}$ consistent with those found in section 2.1 , we need not also require conservation of energy.

The contact surface separating the two shocked regions has to be at rest with respect to the gas, hence $P_{1}^{\prime}=P_{2}^{\prime}$. By this condition the main parameters $\rho_{1}, U_{1}$ and $\rho_{2}, U_{2}$ are linked together. Note that the temperature will not be continuous across the contact surface.

From eqs. $(8 \mathrm{a}, \mathrm{b})$ it follows that

$$
\begin{aligned}
& S_{1}-U_{1}=-\frac{U_{1}}{1-r_{1}} \\
& S_{2}-U_{2}=-\frac{U_{2}}{1-r_{2}},
\end{aligned}
$$

while eqs. $(9 \mathrm{a}, \mathrm{b})$ become

$$
\begin{aligned}
& P_{1}^{\prime}=\frac{\rho_{1} U_{1}^{2}}{1-r_{1}} \\
& P_{2}^{\prime}=\frac{\rho_{2} U_{2}^{2}}{1-r_{2}} .
\end{aligned}
$$

Equating $P_{1}^{\prime}$ and $P_{2}^{\prime}$ gives

$$
\begin{gathered}
\frac{U_{2}}{U_{1}}=-\left(\frac{\rho_{1}}{\rho_{2}} \frac{1-r_{2}}{1-r_{1}}\right)^{\frac{1}{2}}=-q, \\
U_{1}=-\frac{U_{2}-U_{1}}{1+q} \\
U_{2}=+\frac{U_{2}-U_{1}}{1+1 / q} .
\end{gathered}
$$

It is now possible to express some parameters in terms of the collision velocity $U_{2}-U_{1}$. Substitution of eqs. $(13 \mathrm{a}, \mathrm{b})$ into eqs. $(10 \mathrm{a}, \mathrm{b})$ and $(11 \mathrm{a}, \mathrm{b})$ yields

$$
\begin{gathered}
S_{1}-U_{1}=+\frac{U_{2}-U_{1}}{\left(1-r_{1}\right)(1+q)} \\
S_{2}-U_{2}=-\frac{U_{2}-U_{1}}{\left(1-r_{2}\right)(1+1 / q)} \\
P_{1}^{\prime}=P_{2}^{\prime}=\frac{\rho_{1}\left(U_{2}-U_{1}\right)^{2}}{\left(1-r_{1}\right)(1+q)^{2}}=\frac{\rho_{2}\left(U_{2}-U_{1}\right)^{2}}{\left(1-r_{2}\right)(1+1 / q)^{2}} .
\end{gathered}
$$

With aid of the ideal gas law $T=P \mu H / k \rho$, where $H$ is the proton mass, the temperatures are found

$$
\begin{aligned}
& T_{1}^{\prime}=\frac{\mu_{1}^{\prime} H}{k} \frac{r_{1}}{1-r_{1}} \frac{\left(U_{2}-U_{1}\right)^{2}}{(1+q)^{2}} \\
& T_{2}^{\prime}=\frac{\mu_{2}^{\prime} H}{k} \frac{r_{2}}{1-r_{2}} \frac{\left(U_{2}-U_{1}\right)^{2}}{(1+1 / q)^{2}} .
\end{aligned}
$$

The consistency condition on $r_{1}$ and $r_{2}$ is that the values used in eqs. (9)-(16) satisfy eq. (6) with its input parameter $V_{1}$ replaced by $S_{1}-U_{1}$ for the first shock and $-\left(S_{2}-U_{2}\right)$ for the second shock.

Thus, in the strong shock approximation, for a given collision velocity $U_{2}-U_{1}$ all solutions are related to 
TABLE 3

Values of some parameters connected with the two shocks created by a gas collision at $U_{2}-U_{1}=500 \mathrm{~km} / \mathrm{sec}$, for different values of $\rho_{1} / \rho_{2}$. The quantity $\left|U_{1}\right|$ denotes the velocity of the contact surface towards the galactic plane. Solutions for values of $\rho_{1} / \rho_{2}<1$ can be obtained by reversing the indices 1 and 2 .

\begin{tabular}{c|c|c|c|c|c|c|c|c|c|c}
\hline$\rho_{1} / \rho_{2}$ & $r_{1}$ & $r_{2}$ & $\begin{array}{c}\left|S_{1}-U_{1}\right| \\
(\mathrm{km} / \mathrm{sec})\end{array}$ & $\begin{array}{c}\left|S_{2}-U_{2}\right| \\
(\mathrm{km} / \mathrm{sec})\end{array}$ & $\begin{array}{c}T_{1}{ }^{\prime} \\
\left({ }^{\circ} \mathrm{K}\right)\end{array}$ & $\begin{array}{c}T_{2}{ }^{\prime} \\
\left({ }^{\circ} \mathrm{K}\right)\end{array}$ & $\begin{array}{c}\left|U_{1}\right| \\
(\mathrm{km} / \mathrm{sec})\end{array}$ & $\begin{array}{c}\left|U_{2}\right| \\
(\mathrm{km} / \mathrm{sec})\end{array}$ & $\begin{array}{c}\left|S_{1}\right| \\
(\mathrm{km} / \mathrm{sec})\end{array}$ & $\begin{array}{c}\left|S_{2}\right| \\
(\mathrm{km} / \mathrm{sec})\end{array}$ \\
\hline 64 & 0.098 & 0.248 & 67 & 585 & $2.98 \times 10^{4}$ & $4.82 \times 10^{6}$ & 60 & 440 & 7 & 145 \\
25 & 0.187 & 0.247 & 106 & 550 & $1.29 \times 10^{5}$ & $4.25 \times 10^{6}$ & 86 & 414 & 20 & 136 \\
16 & 0.203 & 0.247 & 129 & 528 & $2.01 \times 10^{5}$ & $3.92 \times 10^{6}$ & 102 & 398 & 27 & 130 \\
9 & 0.220 & 0.247 & 163 & 495 & $3.42 \times 10^{5}$ & $3.46 \times 10^{6}$ & 127 & 373 & 36 & 122 \\
4 & 0.233 & 0.246 & 219 & 441 & $6.46 \times 10^{5}$ & $2.73 \times 10^{6}$ & 168 & 332 & 51 & 109 \\
1 & 0.242 & 0.242 & 330 & 330 & $1.52 \times 10^{6}$ & $1.52 \times 10^{6}$ & 250 & 250 & 80 & 80 \\
\hline
\end{tabular}

one single parameter $\rho_{1} / \rho_{2}$, as long as the value of $V_{i}$ remains fixed. In table 3 numerical values have been obtained from eqs. (13)-(16) for the case $U_{2}-U_{1}=$ $500 \mathrm{~km} / \mathrm{sec}$, the consistency of $r_{1}$ and $r_{2}$ being guaranteed by the use of table 2 . This implies that the same entries have been taken as in that table, in particular $\mu_{1}^{\prime}=\mu_{2}^{\prime}=0.625, V_{i}=50 \mathrm{~km} / \mathrm{sec}$, regardless of the values of $T_{1}{ }^{\prime}$ and $T_{2}{ }^{\prime}$. Note that always $U_{1}<0, U_{2}>0$, $S_{1}-U_{1}>0, S_{2}-U_{2}<0$.

\section{Radiation losses}

The principal energy losses of colliding gas clouds will be radiative. Although hydrogen and helium dominate in abundance, the energy losses are mainly caused by collisional transitions in the heavier ions. Tabular values by PotтAsch (1965) assume H, He, O, $\mathrm{C}, \mathrm{Mg}, \mathrm{Si}$ and Ne abundances of $1: 10^{-1}: 10^{-3}: 10^{-3.3}$ : $10^{-4}: 10^{-4}: 10^{-4}$ and ionization equilibria according to House (1964).

In table 4 is listed the time it takes the ionized gas to cool from a given temperature down to $20000{ }^{\circ} \mathrm{K}$ at either constant density or constant pressure. The quantity $L^{*}$ tabulated by Pottasch is related to the power $L$ radiated per unit volume by

$$
L=n_{\mathrm{H}}^{\prime} n_{\mathrm{e}}^{\prime} L^{*}=\left\{\begin{array}{l}
-\frac{3}{2} n^{\prime} k\left(\frac{\partial T^{\prime}}{\partial t}\right)_{\rho^{\prime}} \\
-\frac{5}{2} n^{\prime} k\left(\frac{\partial T^{\prime}}{\partial t}\right)_{P^{\prime}} .
\end{array}\right.
$$

In eqs. $(17 \mathrm{a}, \mathrm{b}) L$ has been equated to the rate of change of the internal energy at resp. constant volume and constant pressure. In order to account for ionization losses, in both equations a term $-\partial\left(\frac{1}{2} \rho^{\prime} V_{i}^{2}\right) / \partial t$ should have been added to the right-hand side. Again no serious error is made by taking $V_{i}$ constant as long as the gases are essentially ionized. Evidently it is also permitted to put $n_{\mathrm{H}}^{\prime}=n_{\mathrm{e}}^{\prime}=\frac{1}{2} n^{\prime}$. We tabulate therefore

$$
\begin{aligned}
& t_{\rho}^{*}\left(T^{\prime}\right)=n_{\mathrm{H}}^{\prime} t_{\rho}\left(T^{\prime}\right)=3 k \int_{20000}^{T^{\prime}} \frac{\mathrm{d} T}{L^{*}(T)} \\
& t_{P}^{*}\left(T^{\prime}\right)=n_{\mathrm{H}}^{\prime}\left(T^{\prime}\right) t_{P}\left(T^{\prime}\right)=5 k \int_{20000}^{T^{\prime}} \frac{T}{T^{\prime}} \frac{\mathrm{d} T}{L^{*}(T)} .
\end{aligned}
$$

TABLE 4

\begin{tabular}{r|c|c|c}
\multicolumn{4}{|c}{ Cooling times } \\
\hline $\begin{array}{c}T^{\prime} \\
\left({ }^{\circ} \mathrm{K}\right)\end{array}$ & $\begin{array}{c}L^{*} \\
\left(10^{-23} \mathrm{erg} \mathrm{cm}^{3} \mathrm{sec}^{-1}\right)\end{array}$ & $\begin{array}{c}t_{\rho}{ }^{*} \\
\left(\mathrm{~cm}^{-3}\right)\end{array}$ & $\begin{array}{c}t_{P}{ }^{*} \\
\left(\mathrm{y} \mathrm{cm}^{-3}\right)\end{array}$ \\
\hline 20000 & 9.9 & 0 & 0 \\
25000 & 6.8 & $8.1 \times 10^{2}$ & $1.2 \times 10^{3}$ \\
30000 & 12 & $1.8 \times 10^{3}$ & $2.2 \times 10^{3}$ \\
40000 & 18 & $2.8 \times 10^{3}$ & $2.9 \times 10^{3}$ \\
65000 & 57 & $4.0 \times 10^{3}$ & $3.1 \times 10^{3}$ \\
80000 & 65 & $4.3 \times 10^{3}$ & $3.1 \times 10^{3}$ \\
100000 & 50 & $4.8 \times 10^{3}$ & $3.2 \times 10^{3}$ \\
125000 & 57 & $5.4 \times 10^{3}$ & $3.4 \times 10^{3}$ \\
160000 & 57 & $6.2 \times 10^{3}$ & $3.8 \times 10^{3}$ \\
200000 & 65 & $7.0 \times 10^{3}$ & $4.3 \times 10^{3}$ \\
300000 & 32 & $1.0 \times 10^{4}$ & $7.4 \times 10^{3}$ \\
400000 & 17 & $1.6 \times 10^{4}$ & $1.5 \times 10^{4}$ \\
650000 & 12 & $3.9 \times 10^{4}$ & $4.2 \times 10^{4}$ \\
800000 & 9.3 & $5.8 \times 10^{4}$ & $6.2 \times 10^{4}$ \\
1000000 & 7.5 & $8.9 \times 10^{4}$ & $9.7 \times 10^{4}$ \\
2000000 & 4.4 & $3.3 \times 10^{5}$ & $3.6 \times 10^{5}$ \\
4000000 & 2.2 & $1.2 \times 10^{6}$ & $1.4 \times 10^{6}$ \\
\hline
\end{tabular}

However, it is seen that the cooling times do not depend very much on the circumstances under which the cooling takes place. As a representative value of a cooling time the mean of $t_{\rho}^{*}$ and $t_{P}^{*}$ can be taken.

It should be noticed that cooling during the shock is always negligible, as it involves collisional excitation and the shock is only a mean free path thick. 
In section 4 it is assumed that after having cooled down to $20000{ }^{\circ} \mathrm{K}$ the gas rapidly recombines yielding observable neutral hydrogen.

\section{Provisional conclusions}

A combination of the results of the preceding sections seems hardly rewarding, as section 2.2 deals only with steady flow, whereas section 3 considers a timedependent problem. To overcome this lack of consistency, consider the figures 3 and 4 . They are $t-x$ diagrams commonly used in hydrodynamics. Figure 3 represents the steady flow corresponding to the bottom line of table $3\left(\rho_{1}=\rho_{2}\right.$, which makes the solution symmetric). If the cooling estimates of section 3 are

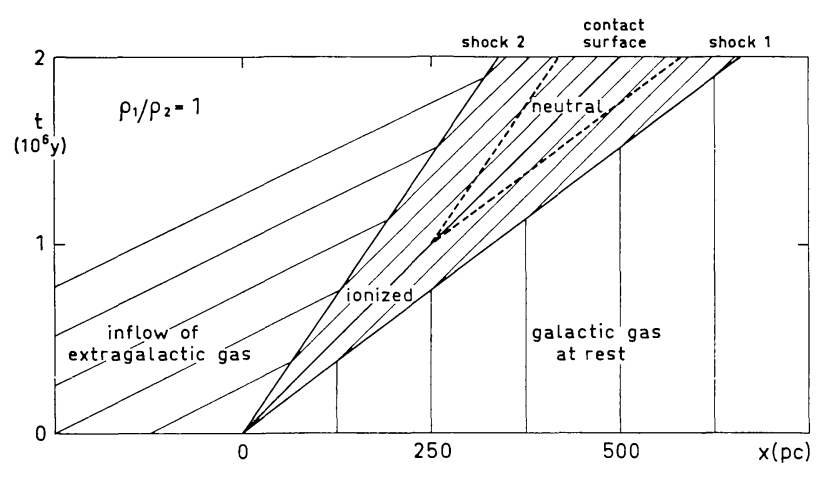

Figure 3. Flow diagram of the steady symmetric solution for a collision velocity of $500 \mathrm{~km} / \mathrm{sec}$. The quantity $x$ denotes pathlength, $t$ denotes time. The shocks are assumed to originate at $t=0, x=0$. Thin lines are streamlines. The broken lines connect all volume elements that have been shocked one cooling time ago. The cooling time applies to a gas with $n_{\mathrm{H}}{ }^{\prime}=0.2 \mathrm{~cm}^{-3}$.

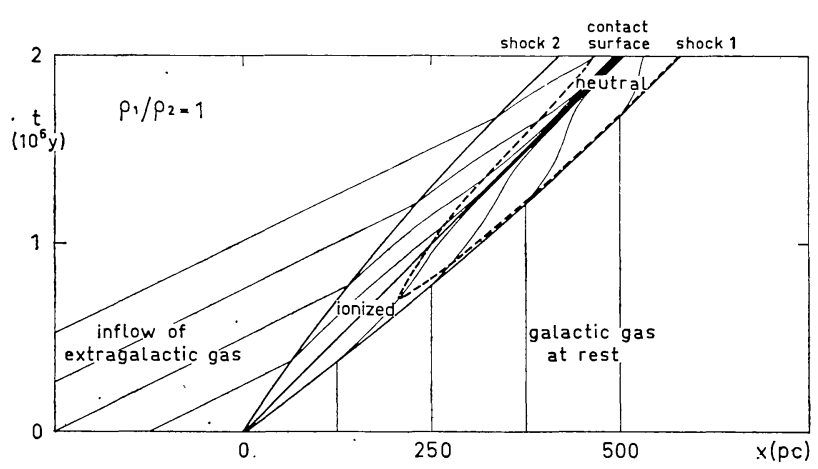

Figure 4. Sketch of the symmetric solution with cooling contraction included. The initial conditions are the same as in figure 3. In the shape of the recombination front (broken curve) the increase of the galactic gas density towards the galactic plane has been accounted for. applied without change, neutral gas would be found in the area between the broken lines.

This representation is far from correct, because it neglects the change in the flow pattern caused by the cooling of the gas. We have been more realistic in figure 4 , which applies to the same initial conditions but takes into account the cooling contraction of the gas. The contraction will cause the shocks to slow down with respect to the gas they are penetrating, until they move with the same speed as the contact surface. The shock strength will decrease and so will the temperature of the ionized gas, hence cooling behind the shocks occurs sooner.

An even more important factor in shortening the cooling times is the increase of the galactic gas density towards the galactic plane. Both effects have been qualitatively taken into account in the shape of the recombination front in figure 4. However, the inhomogeneity of the galactic gas would also bend all streamlines in figure 4 to the left, as follows from the conservation of momentum. This effect has not yet been estimated and is therefore not shown in figure 4 . A good model probably also should include the finite extent of the clouds in all dimensions. The relevant hydrodynamic problems have been considered by Dr. Gross of Columbia University, who spent part of the year in Leiden, and Van Leer. In anticipation of the results of this work, the model sketched in figure 4 will serve as a basis for some provisional comparisons with observations.

Consider only the first shock (in the galactic gas) and assume that its deceleration from $330 \mathrm{pc} / 10^{6} \mathrm{y}$ to 250 $\mathrm{pc} / 10^{6} \mathrm{y}$ takes place uniformly in two times the cooling time at $t=0$, which equals $2 \times 0.20 \times 10^{6} \mathrm{y} \mathrm{cm}^{-3} / n_{\mathrm{H}}^{\prime}$. Since $r_{1} \approx 1 / 4$, we have $n_{\mathrm{H}}^{\prime} \approx 4 n_{\mathrm{H}}$. After one cooling time the shock has swept up $0.20 \times 10^{6} \mathrm{y} \mathrm{cm}^{-3} / 4 n_{\mathrm{H}} \times$ $310 \mathrm{pc} / 10^{6} \mathrm{y} \times n_{\mathrm{H}}=4.8 \times 10^{19}$ hydrogen atoms $/ \mathrm{cm}^{2}$, the greater part of which is neutral again. This value is well within the range of the observed surface densities. The shock has traveled by then over a distance of $310 \mathrm{pc}$, if $n_{\mathrm{H}}^{\prime}$ is rather arbitrarily taken to be 0.2 , as in figures 3 and 4 . Note that the amount of gas swept up is more certain than this distance, which still contains the estimate $n_{\mathrm{H}}^{\prime}$. If we observe the gas after two cooling times, the surface density and path length amount to respectively $9.0 \times 10^{19} \mathrm{~cm}^{-2}$ and $580 \mathrm{pc}$.

No precise statement can be made about the distance 
of the clouds to the galactic plane, since the height at which the shocks originate is not accurately known, probably less than $1 \mathrm{kpc}$ (see section 2). Oort assumes that the galactic latitude of the clouds is representative for the angle at which the inflow occurs (about $40^{\circ}$ ). If this is true, the first shock will proceed in the negative $z$-direction over distances of $200 \mathrm{pc}$ and $370 \mathrm{pc}$ in respectively one and two million years. Hence the clouds will be observable at heights less than 800 pc. A lower limit cannot yet be given.

Our model does not definitely answer the question which gas actually is seen in the $21-\mathrm{cm}$ line, nor does it quantitatively predict the observed velocities. In figure 4 the neutral extragalactic and galactic gas cannot be distinguished by their velocities, which both are close to $250 \mathrm{~km} / \mathrm{sec}$. But the extragalactic component has a lower surface density than the galactic one, in particular because of the increase of the galactic density as the system reaches smaller galactic heights. This leads to the curious conclusion that the high-velocity features may just be galactic gas propelled and compressed by a high-momentum inflow of extragalactic matter.

\section{Acknowledgements}

The authors wish to thank Professors H. C. van de Hulst and R. Gross for numerous fruitful discussions.

\section{References}

J. M. Burgers, 1951, Problems of Cosmical Aerodynamics (Central Air Documents Office, U.S.A.) chapter 8

L. L. House, 1964, Ap. J. Suppl. 8307

A. N. M. Hulsbosch and E. Raimond, 1966, Bull. Astr. Inst. Netherlands 18413

J. H. OORT, 1966, Bull. Astr. Inst. Netherlands 18421

S. R. PotTASCH, 1965, Bull. Astr. Inst. Netherlands 187 